\title{
Development and Validation of IBSA Photographic Scale for the Assessment of Neck Laxity
}

This article was published in the following Dove Press journal:

Clinical, Cosmetic and Investigational Dermatology

\author{
Antonello Tateo' \\ Gabriel Siquier-Dameto $\mathbb{D D}^{2-4}$ \\ Ofir Artzi iD ${ }^{5}$ \\ Dalvi Humzah iD ${ }^{6}$ \\ Beatriz Molina ${ }^{7}$ \\ Ravi Jain ${ }^{6}$ \\ Arturo Lanzarotti ${ }^{8}$ \\ Malika Laouedj ${ }^{9}$ \\ Nicolas Dapis ${ }^{10}$ \\ Gilberto Bellia (D) I \\ 'Private Practice, Milan, Italy; ${ }^{2}$ Private \\ Practice, Amsterdam, the Netherlands; \\ ${ }^{3}$ Private Practice, Mallorca, Spain; \\ ${ }^{4}$ Research Group of Clinical Anatomy, \\ Embryology and Neuroscience \\ (NEOMA), Department of Medical \\ Sciences, Universitat de Girona (UdG), \\ Girona, Spain; ${ }^{5}$ Department of \\ Dermatology, Tel Aviv Sourasky Medical \\ Center, Tel Aviv, Israel; ${ }^{6}$ Private Practice, \\ London, UK; ${ }^{7}$ Private Practice, Bristol, \\ UK; ${ }^{8}$ Research \& Development, IBSA \\ Institut Biochimique SA, Lugano, \\ Switzerland; ${ }^{9}$ Clinical Operations, \\ Quantificare SA, Valbonne, France; \\ ${ }^{10}$ Business Development, Quantificare \\ SA, Valbonne, France; "Medical Affair, \\ IBSA Farmaceutici Italia, Lodi, Italy
}

Objective: To describe the development and validation of the 5-grade photographic IBSA Neck Laxity Scale.

Methods: The scale was developed from 2 real images, which led to the creation of 5 morphed images, representing different degrees of severity of laxity of the neck. For validation, a set of 50 images ( 25 real and 25 morphed) was created and sent for evaluation to 6 trained raters (physicians) in 2 rounds, 1 month apart. Raters had to assess each image according to the 5-image scale. Inter-rater and intra-rater reliability in both rounds was evaluated.

Results: As to intra-rater reliability, single rater kappa scores between 0.69 and 0.87 , and a global kappa score of 0.78 were observed. Inter-rater agreement was measured by means of the intra-class correlation coefficient and scores higher than 0.85 were reported, indicating excellent reliability.

Conclusion: IBSA Neck Laxity Scale is a validated and reliable scale.

Keywords: photographic scale, neck, laxity, aesthetic medicine, scale validation, scale development

\section{Introduction}

Being a particularly exposed area with early extrinsic and intrinsic evident aging textural changes, the anatomical region of the neck represents the target of many aesthetic rejuvenation procedures. As the years go by, wrinkled and sagging skin, platysmal bands and Venus rings, and/or blunting of the cervicomental angle begin to appear. ${ }^{1}$

The current emerging need is to have a younger overall appearance, a fresh look that includes all the most visible body districts, the neck being among them, and this growing demand requires physicians to be prepared to recognize different clinical scenarios, to suggest and perform the most appropriate treatment.

A youthful neck is characterized by an acute cervico-mental angle, a firm and well-defined jawline, a smooth skin with minimal melanin or vascular lesions, no horizontal or vertical neck lines, absence of any platysmal band, no visible submandibular gland, and no hypertrophic masseter muscles. ${ }^{2}$

The neck region is characterized by the passage of crucial vascular and nervous structures for the entire craniofacial district and by a sophisticated muscularaponeurotic network. From an anatomical point of view, different layers can be recognized, therefore from the most superficial to the deepest it is possible to identify the skin, the subcutaneous tissue, the superficial musculofacial plane, and
Correspondence: Gilberto Bellia IBSA Farmaceutici Italia, Lodi, LO, Italy Email gilberto.bellia@ibsa.it 
finally the sub-platysmal structures (Figure 1$){ }^{2}$ The ageing process involves all these structures with different mechanisms.

The cutaneous layer of the neck consists of a relatively thin epidermis and dermis which have to bear several tensile and compressive stresses; moreover, the frequent movements of the neck in anterior-posterior and lateral direction which are made possible by the contraction of the underlying platysma can be the cause of the so-called "necklace lines". These horizontal neck wrinkles are linear depressions in the anterior side of the neck. Whereas facial wrinkles are certainly caused by skin aging, horizontal neck wrinkles are not rarely seen in children and young adults and age-related skin laxity can make them even worse: repeated bending of the neck to look at cell phones, tablets, or books could also lead to wrinkle development in younger subjects. However, age-related skin laxity can make these wrinkles more evident. ${ }^{3}$

Moreover, the skin of the neck often shows a significant extrinsic photoaging, characterized by increased epidermal thickness, degeneration of collagen and elastin, and deposit of loops of elastotic collagen in the deep dermis. ${ }^{2,3}$ Subcutaneous or adipose tissue is located deep into the epidermal-dermal plane, and its amount greatly varies among individuals.
The cervical platysmal layer consists of wide strapshaped skeletal muscles which span from the dermal attachments along the mandibular border to the clavicle. The superficial layer of the deep cervical fascia invests the cervical platysma and it stretches upwards where it is known as the superficial-muscular aponeurotic system (SMAS). With age, the retaining ligaments that maintain the free medial edges anatomically close to the deep cervical fascia become weaker, and medial edges descend leading to platysma bands. ${ }^{4}$ With muscle flaccidity and atrophy typical of aging, platysma bands can worsen cervical laxity and make it more evident, resulting in a sagging, adynamic, and obtuse neck. ${ }^{2}$

As the subcutaneous fullness of the mandible recedes, the fat of the jowl, which was previously concealed by the surrounding soft tissues, is revealed. Ptosis of unsupported skin, coupled with the downward pull of platysma muscle, leads to the development of the characteristic "turkey neck" deformity. In addition, contraction of platysma muscle, caused in part by the need to support the deeper neck and floor of oral structures, gives rise to vertical fibrous bands on the neck, whereas laxity in the overlying skin can create horizontal rhytides. As aging progresses, the hyoid bone and larynx gradually descend, resulting in loss or blunting of the cervicomental angle. ${ }^{5}$

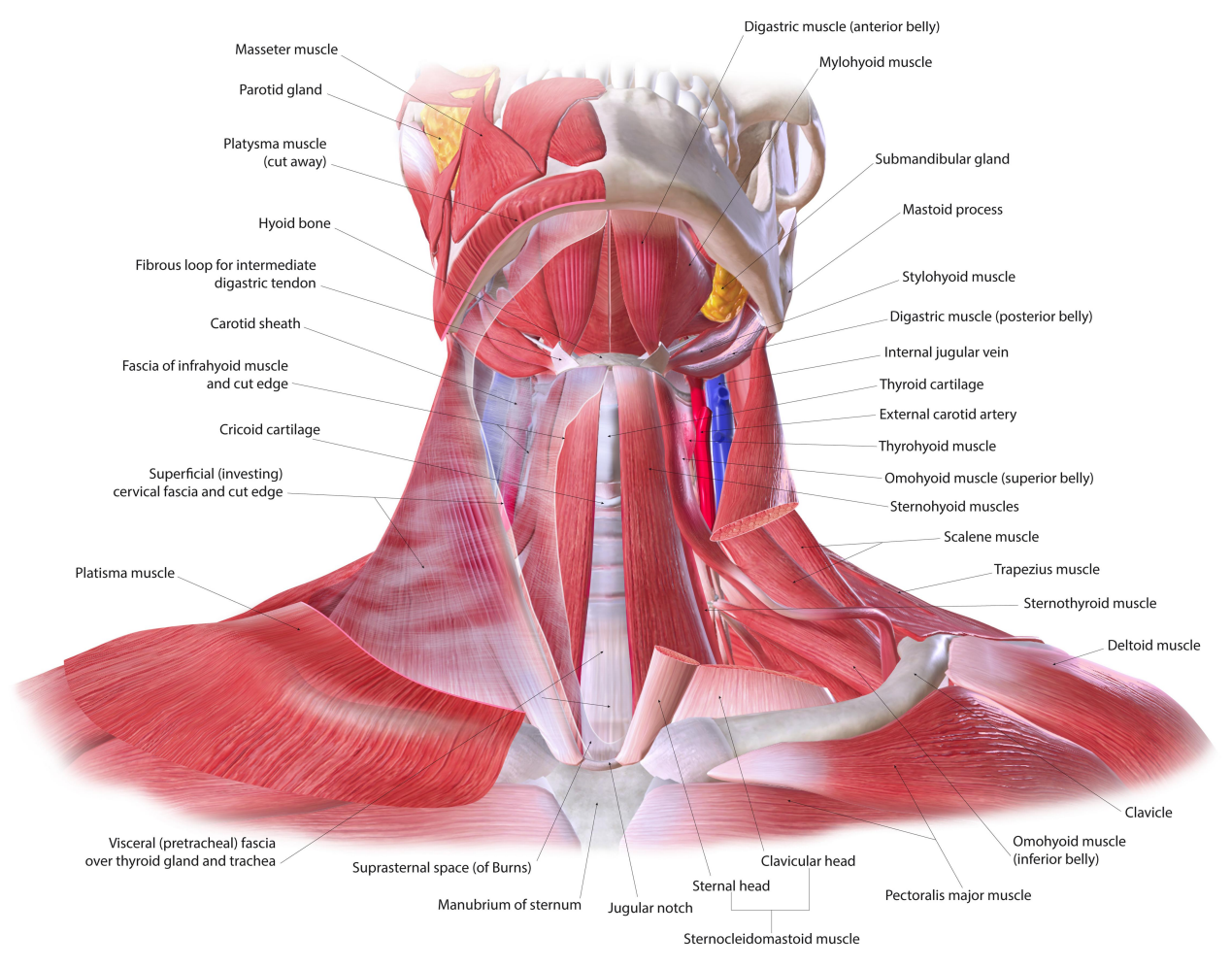

Figure I Neck anatomy. 
Finally, the deep plane includes sub-platysmal fat, the anterior belly of the digastric muscles, and submandibular glands that can become ptotic or hypertrophic with ageing, leading to a visible bulge that disrupts the planar, smooth surface of a youthful-appearing neck. ${ }^{4}$

Neck rejuvenation treatments range from minimally or non-invasive methods to invasive surgical techniques: poor texture, fine lines, dyschromia and photoaging can be improved by fractional nonablative and ablative lasers, as well as ablative fractional radiofrequency devices; several injectables such as botox can help improve the appearance of the neck and jawline, and soften the appearance of neck banding secondary to muscle action; hyaluronic acid (HA) fillers are widely known as a good solution to treat horizontal neck wrinkles and can be used to sculpt the jawline and create improved contours and balance; excessive preplatysmal fat can be addressed by suction-assisted lipoplasty when skin elasticity is fairly preserved, otherwise neck lift is a better option. ${ }^{6,7}$

Obviously, the most invasive treatments pose a series of problems related to safety, as the anatomical region of the neck is both a pivotal passageway and the location of important vascular, nervous, and glandular structures; moreover, being the skin of this region particularly thin, fibrotic and scarring outcomes may represent a complication of invasive treatments.

The choice of one type of intervention rather than another depends on a series of variables closely linked to patient's characteristics. In case of severe tissue deterioration and laxity, surgery - despite its possible complications - is usually the best option for a good aesthetic result. On the other hand, there are a number of intermediate stages in which the use of the filler could be useful and resolving. Currently, the discrimination of individual cases is a matter of personal assessment of physicians, their experience and mere aesthetic judgement. Hence, it is not always easy for clinicians to assess what is the best intervention to implement.

In order to better identify individuals who could benefit more from one treatment than another, and to help doctors in their therapeutic choices, IBSA has designed a photographic scale, validated by physicians for their peers, which consists of 5 grades that differ according to the laxity of neck tissues. The objective is to help physicians overcome some clinical dilemmas by providing them with an objective, clear and easy-to-use tool that allows a quick initial assessment of the patient's situation. According to Italian law, this scale validation was exempt from ethic committee approval because no human beings have been involved. This scale development and validation was conducted according to the ethics of the Declaration of Helsinki. The information and data were generated, recorded, documented, and processed in accordance with a specific procedure, based on the International Council for Harmonization of Technical Requirements for Pharmaceuticals for Human Use (ICH) Good Clinical Practice (GCP). All validators signed an informed consent form at the time of the enrolment in order to collect the clinical data. The process that led to the development and validation of this innovative photographic tool is the topic of this paper.

\section{Methods}

\section{Scale Development}

The scale was developed and validated following a method already used in photonumeric scales adopted for other parts of the body. The methodology which was already tested was then customized for the purposes of the scale here described (Figure 2). ${ }^{8-11}$

A team of three experts (ie a plastic surgeon, the scientific responsible for dermoaestetics at IBSA and the responsible for research and development of QuantifiCare) selected two photographs from the database of a clinician with the aim of identifying two images, one representative of the neck with a healthy aspect and one of the most severe degree of laxity, but still considered eligible for filler treatment. This last photograph was selected to represent a borderline stage, beyond which the use of the filler would not be recommendable as surgery is currently the only option available.

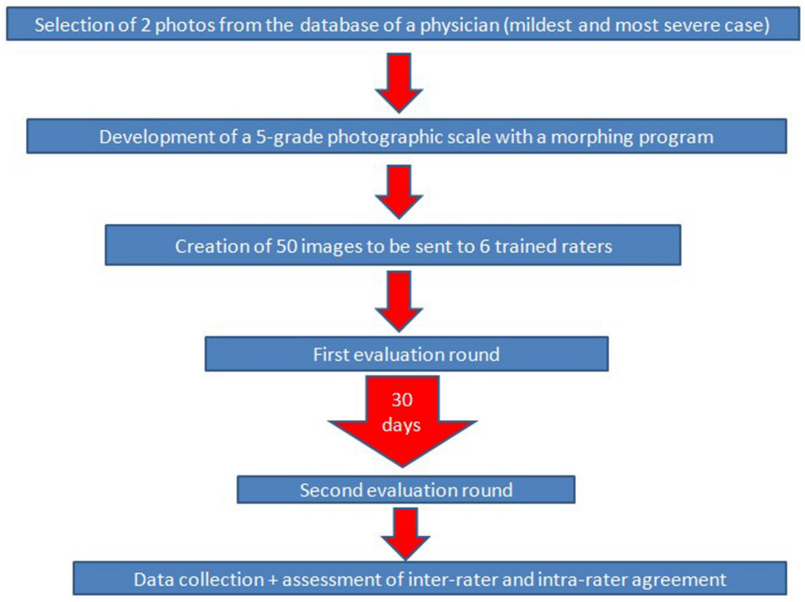

Figure 2 Algorithm of development and assessment of IBSA Neck Laxity Scale. 
A total of 50 photos of necks of patients between 40 and 79 years of age were chosen for this selection. Once this first selection was made, QuantifiCare in close interaction and collaboration with the 3 experts involved created a photographic scale with a morphing program. This scale is composed of 5 images that correspond to different degrees of severity of neck laxity (Figure 3 ). The images show the frontal aspect of the neck, from the lower edge of the mandible to the upper edge of the clavicle, the chin is in the rest position. By taking into account the tone and degree of laxity of the tissues, the 5 grades are identified and as follows:

Grade 1: Normal trophism of the tissues of the neck.

Grade 2: Mild laxity of the tissues of the neck.

Grade 3: Moderate laxity of the tissues of the neck.

Grade 4: Moderate to severe laxity of the tissues of the neck.

Grade 5: Severe laxity of the tissues of the neck.

Subsequently, a set of 50 photographs (25 real and 25 morphed) was created for the validation of the scale by 6 selected raters. Before the presentation of this set of images to the 6 validators and their training on the software, in order to carry out a further and final assessment of the grades and their corresponding descriptors, the 5 morphed images were presented to the raters by means of a ranking exercise during which the 5 selected Grades plus a set of other non-selected morphed images were presented: during this test, validators were only given the descriptors and they had to match the image that better represented the condition described. They had to perform this ranking exercise twice with a break of a week between the two sessions. Validators confirmed the scale which was previously developed.

\section{Software Characteristics}

The software was developed and validated by following GAMP5 methodology, compliant to 21 CFR part 11, it used pseudonymized photographic data sorted in a folder architecture to generate first a Microsoft Access Database file, and finally a Comma Separated Value file. Photographic data were acquired using a Phase one XF IQ150 camera.

As to software, Windows 10 and DirectX 9 or superior was required; as to hardware, a minimum of $2 \mathrm{~GB}$ of free space on the hard drive, and 4GB of RAM memory were required, along with a screen resolution of at least $1024 \times 768$.

The software was divided by page, each containing 1 photo for evaluation. For each page, the user had the possibility to give a score, before submitting it to go to the next page.

As to user requirements, a Webex training session was scheduled. During this initial phase, experts were presented a demo of the tool to ensure the proper evaluation

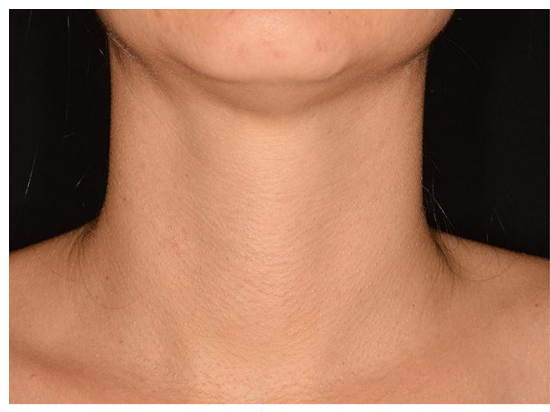

A

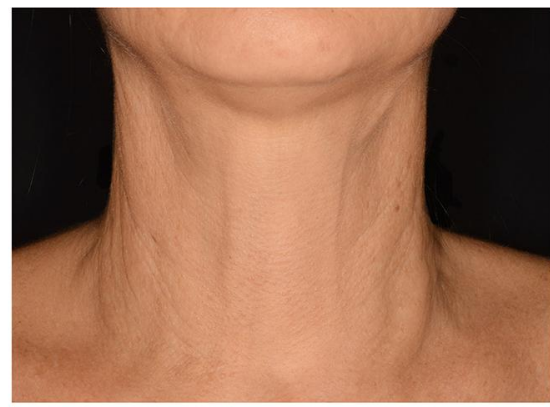

B

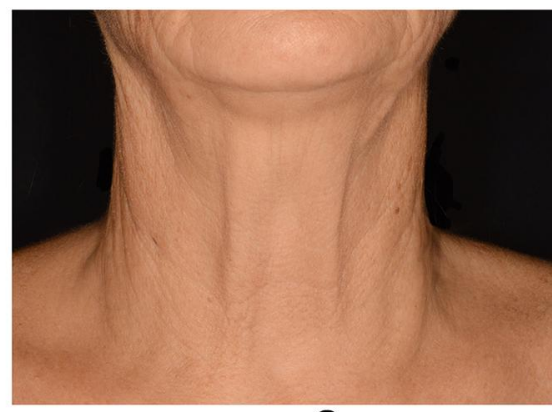

C

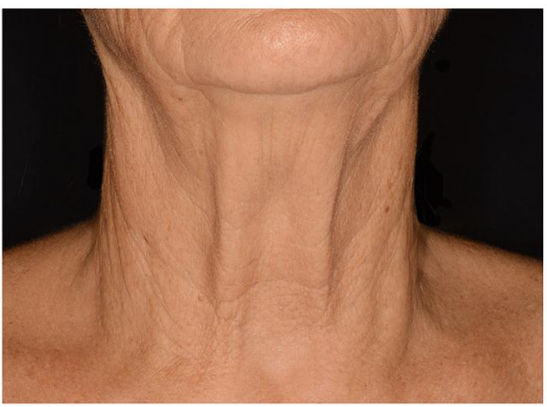

D

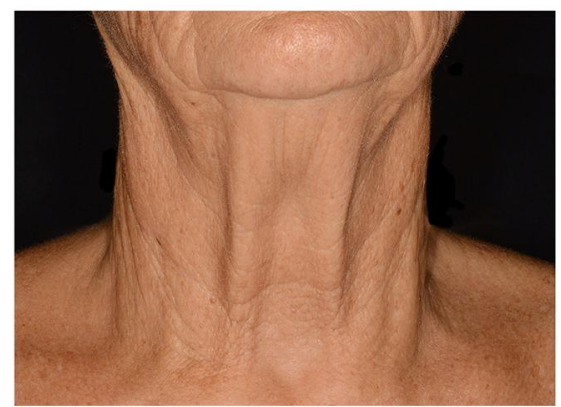

E

Figure 3 The 5-grade IBSA Neck Laxity Scale. (A) Grade I: normal trophism of the tissues of the neck. (B) Grade 2: mild laxity of the tissues of the neck. (C) Grade 3: moderate laxity of the tissues of the neck. (D) Grade 4: moderate to severe laxity of the tissues of the neck. (E) Grade 5: severe laxity of the tissues of the neck. 
of the system, and that no questions would arise during the rating. Validators were given the possibility to restart and go through the training module any time.

All users were given a unique login for correct authentication. Five score options were given (Grade 1, Grade 2, Grade 3, Grade 4, and Grade 5), and experts could only select one. The application displayed one photo per patient, in the center of the window, image size was updated while window was resizing, it was possible to enlarge each photo and fullscreen visualization was available by clicking on it. Users were allowed to stop the evaluation any time and resume it later. The application did not allow to go to next subject without having scored the current one; however, it was possible to reevaluate previous patients, and change any of the scores already given.

Assigned scores were saved during navigation among patients, and there was no time limit. Scores were provided to IBSA in a CSV file, along with a report which also contained results as specified for each subject and name of evaluators.

\section{Scale Validation}

The 50 photographs were sent to the 6 raters who had 30 days for completion of each of two evaluation rounds, one month apart. In the two assessments images were the same, but in a different order.

Finally, data were collected by QuantifiCare and subjected to statistical analysis by IBSA to assess responses of the same rater on the same photo (inter-rater evaluation), and scores of the same photo among the different raters (intra-rater evaluation).

\section{Results}

\section{Intra-Rater Reliability (Table I)}

For scale validation, intra-rater reliability between the first and the second evaluation performed by the same expert was calculated using weighted kappa scores with Fleiss-Cohen weights (the evaluation is a rate, while categories are ordinal).

This analysis was performed assessing each expert alone, and considering all the first and the second available evaluations together.

Kappa scores between 0.69 and 0.87 as to expert analysis, and a global kappa score of 0.78 when the 6 expert evaluations were analyzed all together were observed.

These results indicate substantial/almost-perfect agreement between the first and the second evaluation performed on the same image (kappa scores range between 0 and 1;
Table I Intra-Rater Reliability with Weighted Kappa Scores Calculated with Fleiss-Cohen Weights

\begin{tabular}{|l|c|c|c|}
\hline & $\begin{array}{c}\text { Weighted } \\
\text { Kappa }\end{array}$ & $\begin{array}{c}\text { 95\% Lower } \\
\text { Conf. Limit }\end{array}$ & $\begin{array}{c}\text { 95\% Upper } \\
\text { Conf. Limit }\end{array}$ \\
\hline Expert I & 0.7855 & 0.6818 & 0.8891 \\
Expert 2 & 0.8700 & 0.7916 & 0.9484 \\
Expert 3 & 0.7609 & 0.6448 & 0.8769 \\
Expert 4 & 0.8203 & 0.7301 & 0.9106 \\
Expert 5 & 0.6951 & 0.5818 & 0.8085 \\
Expert 6 & 0.7328 & 0.6264 & 0.8391 \\
Total & 0.7815 & 0.7398 & 0.8231 \\
\hline
\end{tabular}

$0.61-0.80$ indicate substantial agreement; $0.81-1.00$ indicate almost-perfect agreement).

\section{Inter-Rater Reliability (Table 2)}

Inter-rater agreement was measured by calculating intra-class correlation coefficient (ICC $[2,1]$ - to be used when all images are rated by the same raters who are assumed to be a random subset of all possible raters) as described by Shrout and Fleiss.

The analysis was performed considering the first and the second evaluation separately, and analyzing the two evaluations together.

These results indicate good/excellent reliability (intraclass correlation coefficient ranges between 0 and 1; values less than 0.5 are indicative of poor reliability, values between 0.5 and 0.75 indicate moderate reliability, values between 0.75 and 0.9 indicate good reliability, and values greater than 0.90 indicate excellent reliability).

\section{Discussion}

The anatomical region of the neck is particularly exposed to environmental insults and it is undoubtedly one of the first to show signs of premature ageing, which makes rejuvenation of this area one of the increasingly desirable targets for an increasing number of patients. Being a particularly delicate body area from an anatomical and structural point of view, the evaluation of aesthetic defects in the skin and underlying tissues is a crucial moment to discriminate between an invasive treatment with all its

Table 2 Overall Inter-Rater Reliability as Described by ShroutFleiss

\begin{tabular}{|l|c|}
\hline & Shrout-Fleiss Reliability - Random Set \\
\hline Evaluation 1 & 0.8582 \\
Evaluation 2 & 0.8820 \\
Total & 0.9132 \\
\hline
\end{tabular}


possible complications and a less invasive kind of intervention than surgery. Hence, the need to provide the aesthetic doctor with a reliable tool for assessment among the different cases.

This scale based on 5 images was developed using digital techniques starting from real photos, it is a simple and immediate aid, which makes it possible to quickly recognize different clinical circumstances. The scale has been validated by doctors who based their assessment on their clinical experience and the positive results of the validation phase highlight its reliability.

The tool here described identifies a deficit in the volume and shape of the neck region due to aging, and each degree is represented by an iconographic and a verbal reference, ie the image and the descriptor, respectively. However, these two indicators are influenced by individual variables which may represent a limitation to the use of this scale. However, an iconographic and verbal classification as the one here presented, never perfectly mirrors, but rather tries to best describe a possible real situation, the rest is entirely up to the physician's clinical instinct and experience. This is precisely the reason why the only way to truly evaluate the advantages of this scale is to see how it performs in a real-life scenario.

\section{Conclusions}

Overall inter and intra-rater data indicate that the scale created is consistent and reliable.

\section{Funding}

This project has been sponsored by IBSA Farmaceutici Italia.

\section{Disclosure}

AL is an employee of IBSA SA Switzerland. ML \& ND are employees of Quantificare SA France. GB is an employee of IBSA Farmaceutici Italia. The authors report no other conflicts of interest in this work.

\section{References}

1. Ellenbogen R, Karlin JV. Visual criteria for success in restoring the youthful neck. Plast Reconstr Surg. 1980;66(6):826-837. doi:10.1097/00006534-198012000-00003

2. Mulhollad RS. Nonexcisional, minimally invasive rejuvenation of the neck. Clin Plast Surg. 2014;41:11-31. doi:10.1016/j.cps.2013.09.002

3. Tseng F, Yu H. Treatment of horizontal neck wrinkles with hyaluronic acid filler: a retrospective case series. Plast Reconstr Surg Glob Open. 2019;7(8). doi:10.1097/GOX.0000000000002366

4. Mejia JD, Nahai FR, Nahai F, et al. Isolated management of the aging Neck. Semin Plast Surg. 2009;23(4):264. doi:10.1055/s-00291242178

5. Coleman RS, Grover R. The anatomy of the aging face: volume loss and changes in 3-dimensional topography. Aesthet Surg J. 2006;26 (1):S4-S9. doi:10.1016/j.asj.2005.09.012

6. Carruthers A, Carruthers J. Cosmetic uses of botulinum exotoxin. Adv Dermatol. 1997;12:325-347.

7. Manstein D, Herron GS, Sink RK, et al. Fractional photothermolysis: a new concept for cutaneous remodeling using microscopic patterns of thermal injury. Lasers Surg Med. 2004;34(5):426-438. doi:10. $1002 / 1 \mathrm{sm} .20048$

8. Jones D, Donofrio L, Hardas B. Development and validation of a photonumeric scale for evaluation of volume deficit of the hand. Dermatol Surg. 2016;42(Suppl 1):S195. doi:10.1097/DSS.000 0000000000850

9. Sykes JM, Carruthers A, Hardas B. Development and validation of a photonumeric scale for assessment of chin retrusion. Dermatol Surg. 2016;42(Suppl 1):S211. doi:10.1097/DSS.0000000000000849

10. Donofrio L, Carruthers A, Hardas B. Development and validation of a photonumeric scale for evaluation of facial skin texture. Dermatol Surg. 2016;42(Suppl 1):S219. doi:10.1097/DSS.0000000000000852

11. Jones D, Carruthers A, Hardas B. Development and validation of a photonumeric scale for evaluation of transverse neck lines. Dermatol Surg. 2016;42(Suppl 1):S235. doi:10.1097/DSS.000000 0000000851
Clinical, Cosmetic and Investigational Dermatology is an international, peer-reviewed, open access, online journal that focuses on the latest clinical and experimental research in all aspects of skin disease and cosmetic interventions. This journal is indexed on CAS.
The manuscript management system is completely online and includes a very quick and fair peer-review system, which is all easy to use. Visit http://www.dovepress.com/testimonials.php to read real quotes from published authors. 